Reprad. Nutr. Dévelop., 1980, 20 (4 B), 1257-1265.

\title{
Influence d'un régime de malnutrition protéique ( 5 p. 100 de gluten) et d'une réalimentation équilibrée (15 p. 100 de caséine) sur les activités de la lipase potentielle, de la lipase-colipase dépendante et de la phospholipase $A_{2}$.
}

\section{Suc pancréatique de rat en croissance}

\author{
par Josiane PROST, M. BOUZIANE, J. BELLEVILLE
}

Laboratoire de Physiologie Animale ef de la Nutrition,

U. E. R. Nutrition, B. P. 138, 21004 Dijon Cedex, France

Summary. Effect of a protein-deficient diet $(5 \mathrm{p} .100$ glufen) and of balanced refeeding (15 p. 100 casein) on potential lipase, colipase-dependent lipase and phospholipase $A_{2}$ activities. II. In the pancreatic juice of the growing rat.

We experimented with a diet resembling a protein-deficient one eaten by man, having a low protein level and proteins of poor biological value.

Malnutrition caused weight loss accompanied by an overall reduction of ingesta. However, the food intake, as compared to animal weight, was the same in the undernourished animals as in the controls.

Bile and pancreatic juice outputs were not altered continuously and regularly but with the poor protein diet, they were lower. Refeeding a balanced dief caused these outputs to increase.

When 0.6 p. 100 of methionine was added to the deficient diet, no significant difference was noted in the outputs of the rats eating the deficient diets.

Enzyme activities in the juice and pancreas varied widely from one day to another during malnutrition and during refeeding.

The mean values of specific activities, which masked these variations, showed that malnutrition did not significantly decrease the phospholipase $A_{2}$ activity in the pancreatic juice, while potential lipase and colipase-dependent lipase activities declined.

Refeeding temporarily stimulated specific phospholipase $A_{2}$ and potential lipase activities the third day; colipase-dependent lipase activity was not activated.

After 2 to 3 weeks of refeeding, the specific activities of the three enzymes were similar to the control values, but were no longer so at the end of refeeding. This would suggest a return to the normal, as shown by the oscillations around the control values.

Protein malnutrition, as refeeding, did not in general cause the same changes in all the enzymes at the same time.

\section{Introduction.}

Quelques travaux, peu nombreux, ont porté sur les répercussions de la malnutrition proféique et de la réalimentation équilibrée, sur le suc pancréatique et son contenu en enzymes digestives. 
Lemire et lber (1967), Barbezat (1967), Robberecht ef al. (1967), Tandon et al. $(1969,1970)$ ont étudié, entre autres, le comportement de la lipase. Ils trouvent dans le suc pancréatique, une diminution de cette activité au cours de la malnutrition protéique ef un rétablissement partiel ou total de celle-ci durant la réalimentation équilibrée. Cependant, Lyman et Wilcox (1963) avaient montré qu'il suffisait de priver l'animal d'un seul acide aminé indispensable pour abaisser fortement les activités enzymatiques dans le suc pancréatique.

Comme l'existence de la colipase n'était pas connue, dans aucune de ces études les activités lipasiques potentielle et colipasique dépendante n'ont été mesurées.

Les résultats des auteurs précédents montrent des divergences quant au comportement de la lipase qui ne dépendent pas uniquement du type de malnutrition protéique utilisée. De même, la réalimentation équilibrée ne donne pas des valeurs toujours convergentes.

Nous avons pensé que cela pouvait être dû au fait que les activités enzymatiques ne diminuent pas linéairement en fonction du temps lors de la malnutrition et de la même façon n'augmentent pas lors de la renutrition : c'est pourquoi dans ce travail, notre étude sera chronologique.

Dans une expérience, nous apportons un supplément de méthionine au régime hypoprotéique, afin de voir s'il existe une sensibilité particulière d'une enzyme vis-àvis de cet acide aminé.

Comme nous effectuons une étude parallèle sur la glande pancréatique (Belleville, Bouziane et Prost, 1980), nous pourrons indiquer si les modifications observées portent sur la synthèse, la rétention ou l'excrétion des enzymes.

\section{Techniques expérimentales.}

Nous ne reviendrons pas sur la composition des régimes, la répartition des animaux, les techniques de dosages (Belleville, Bouziane et Prost, 1980).

Le prélèvement des sucs pancréatiques se fait par double cathétérisme du canal pancréato-biliaire, suivant un processus déjà décrit (Belleville, Prost et Gillet, 1978). Pour chaque régime, tous les 2 ou 3 jours, nous prélevons les sucs pancréatiques de 3 animaux que nous réunissons. Les dosages sont effectués sur ces pools.

\section{Résultats.}

I. - Quantités de nourriture et de protéines ingérées (fig. 1A et B).

1) Quantités de nourriture consommée. - Les animaux carencés, pendant les 5 premiers jours de malnutrition, conservent une prise alimentaire stable, de l'ordre de $10 \mathrm{~g} /$ jour $/$ rat. Puis, cette consommation chute et se stabilise à $6 \mathrm{~g} / \mathrm{jour} / \mathrm{rat}$. Dès le début de la réalimentation équilibrée, les valeurs augmentent rapidement jusqu'à environ $15 \mathrm{~g} / \mathrm{jour} / \mathrm{rat}$, c'est-à-dire des valeurs semblables à celles des animaux témoins.

Si l'on ramène l'ingesta à $100 \mathrm{~g}$ de poids corporel, les malnutris ont au cours des 3 premières semaines des consommations plus faibles que les témoins mais, à la fin de la malnutrition, les consommations deviennent voisines. 


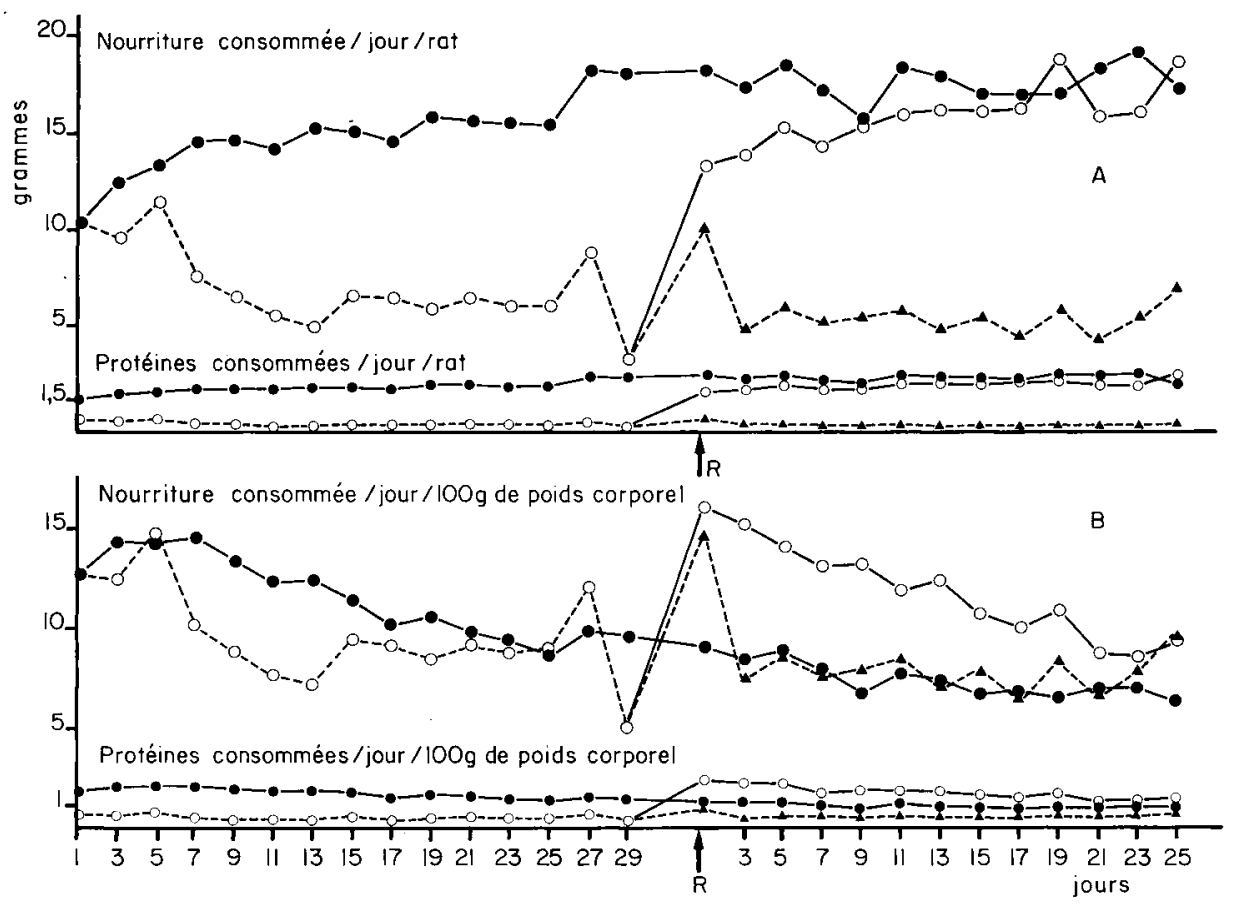

FIG. 1A. - Quantités de nourriture ef de protéines consommées par jour et par rat.

FIG. 1B. - Quantités de nourriture et de protéines consommées par jour ramenées d̀ $100 \mathrm{~g}$ de poids corporel.

$R=$ fin de la malnutrition et début de la réalimentation équilibrée pour les animaux carencés ou le début de l'ajout de méthionine pour les carencés + méthionine.

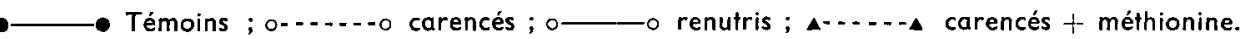

La réalimentation équilibrée augmente considérablement l'ingesta des renutris qui dépasse alors nettement celui des témoins. Cependant, en fin de période de réalimentation, les 2 groupes d'animaux ont des consommations alimentaires $/ 100 \mathrm{~g}$ de poids corporel qui se rapprochent.

2) Quantités de protéines consommées. - Les courbes mettent bien en évidence la faiblesse de la prise protéique chez les animaux carencés qui ne représente que 17 à 20 p. 100 de la consommation protéqque des témoins. La réalimentation équilibrée correspond à un retour vers les valeurs témoins de l'ingesta protéique, ce qui entraîne une surconsommation de protéines $/ 100 \mathrm{~g}$ de poids chez les renutris $(+70 \mathrm{p} .100 \mathrm{par}$ rapport aux témoins), puisque ces animaux conservent un déficit pondéral. Cette différence s'atténue avec le temps, mais elle persistera encore au $27 \mathrm{e}$ jour de réalimentation.

L'enrichissement du régime carencé en méthionine stimule la prise alimentaire, donc protéique, le 1 er jour, mais, très vite, ces consommations retombent à ce qu'elles étaient pendant la carence. 
II. - Débits de suc pancréatique et de bile (fig. 2A, B, C et D).

Les débits de suc pancréatique $(\mathrm{ml} / \mathrm{h})$ augmentent avec l'âge des animaux. Ils sont en général inférieurs chez les animaux carencés ef chez les renutris par rapport aux témoins. L'ajout de méthionine au régime carencé entraîne une importante mais fugace stimulation de ce débit le $3 e$ jour.

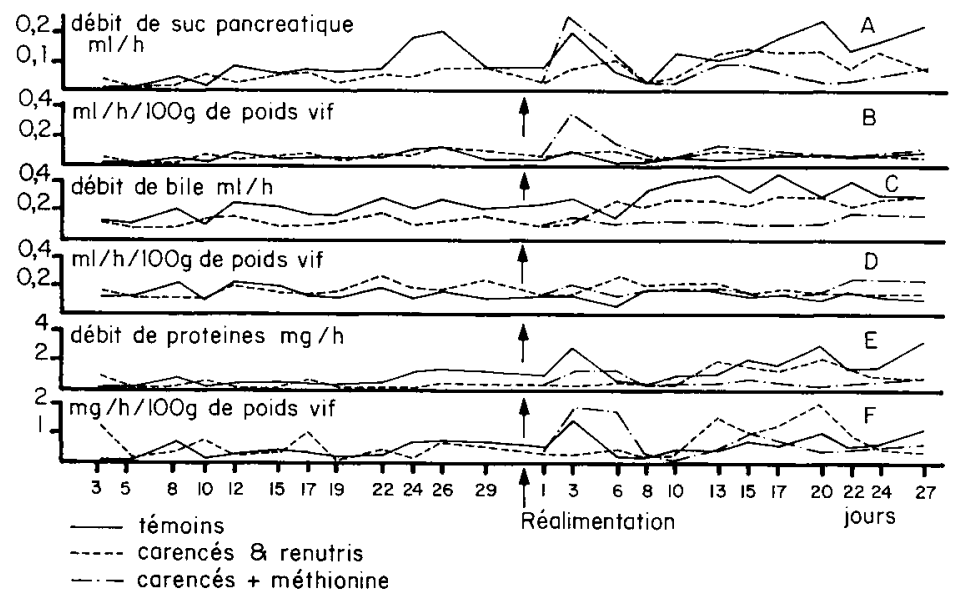

FIG. 2. - Débits de suc pancréatique, de bile, de protéines du suc, donnés pour un rat (A. C. E.) ou rapportés a $100 \mathrm{~g}$ de poids corporel (B. D. F.). La flèche indique la fin de la malnutrition et le début de la réalimentation équilibrée pour les animaux carencés ou le début de l'ajout de méthionine pour les carencés + méthionine.

Lorsque les débits de suc sont exprimés en $\mathrm{ml} / \mathrm{h} / 100 \mathrm{~g}$ de poids, les valeurs deviennent voisines chez les témoins et les carencés, lors de la malnutrition ef chez les témoins et les renutris, lors de la réalimentation.

Les débits de bile $(\mathrm{m} / \mathrm{h})$ sont plus faibles que ceux des témoins chez les carencés, les renutris ef les carencés + méthionine. Ramenés à $100 \mathrm{~g}$ de poids, ces mêmes débits deviennent voisins chez tous les lots d'animaux.

III. - Débits de protéines (fig. 2E, F).

Le débit de protéines $(\mathrm{mg} / \mathrm{h})$ est semblable chez les carencés et les témoins durant la $1^{\text {re }}$ quinzaine de malnutrition puis il devient inférieur aux témoins en fin de période. Lors de la réalimentation, les renutris ef les témoins ont des débits de protéines voisins.

Lorsque ces débits sont exprimés en $\mathrm{mg} / \mathrm{h} / 100 \mathrm{~g}$ de poids, durant toute la malnutrition, carencés et témoins ont des débits semblables, tandis que, pendant la réalimentation, les renutris ont des valeurs supérieures aux témoins.

L'ajout de méthionine au régime carencé ne modifie pas d'une façon significative les résultats obtenus à la fin de la malnutrition. 
IV. - Activités de la lipase potentielle, de la lipase-colipase dépendante, de la phospholipase $A_{2}$.

1) Activités spécifiques (AS) (fig. 3). - Elles montrent de grandes variations d'un dosage à l'autre. La malnutrition, au début, stimule les AS de la lipase potentielle ef surfout de la lipase colipase dépendante.

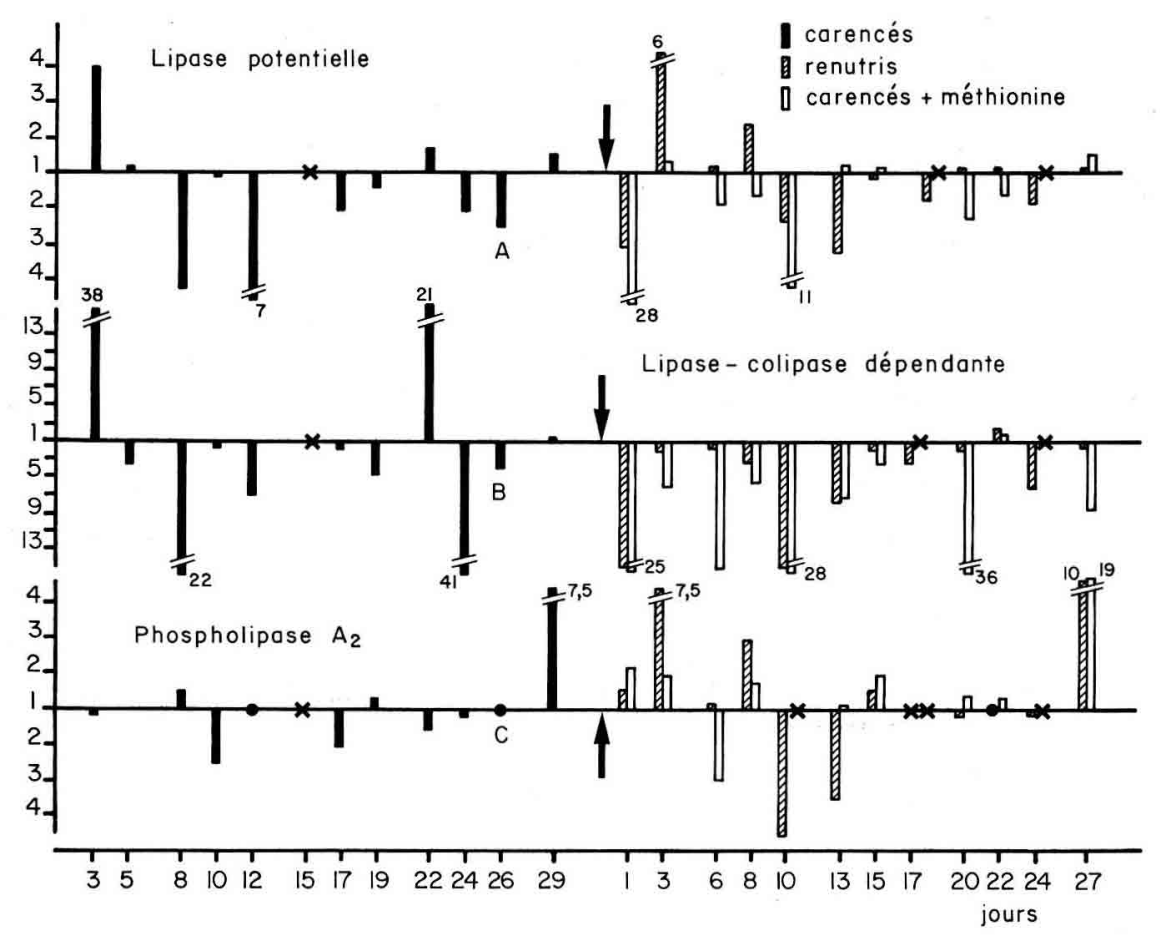

FIG. 3. - Activités spécifiques de la lipase potentielle, de la lipase-colipase dépendante et de la phospholipase $A_{2}$, exprimées en $\mu E q$ de substrat hydrolysé/min/mg de protéine.

La flèche indique la fin de la malnutrition. Les points signifient que la valeur expérimentale est la même que la valeur témoin. Les " $x$ » correspondent à une absence de dosage. La ligne qui marque la base des diagrammes correspond à la valeur obtenue chez les témoins, elle est égale à 1. Les diagrammes expriment les valeurs expérimentales trouvées chez les animaux carencés, renutris ou carencés + méthionine, en fonction des valeurs témoins. Les chiffres placés en ordonnées donnent la valeur de $X$ :

- lorsque le diagramme est situé au-dessus de la ligne de base, ils indiquent que la valeur expérimentale est égale à $X$ fois plus la valeur témoin ;

- lorsque le diagramme est situé en dessous de la ligne de base, ils indiquent que la valeur expérimentale est égale à $X$ fois moins la valeur témoin.

Donc, plus le diagramme est élevé au dessus de la ligne de base, plus la valeur trouvée est supérieure à la valeur témoin et plus la valeur est importante en dessous de la ligne de base, plus la valeur obtenue est inférieure à la valeur témoin.

Nous constatons que ces activités ne s'atténuent pas en fonction de la durée de la malnutrition et, en particulier, celle-ci se termine par des valeurs supérieures pour les 3 AS chez les animaux malnutris. Seule, l'AS de la lipase colipase dépendante est un 
peu diminuée sur l'ensemble de la période (- 1,6 fois par rapport aux témoins, en moyenne). Par contre, les moyennes des AS de la lipase potentielle et de la phospholipase $A_{2}$ ne sont pas significativement abaissées.

Après $24 \mathrm{~h}$ de réalimentation, il y a inhibition des 3 AS mais, il existe une stimulation après 3 jours, pour les AS de la lipase potentielle ef de la phospholipase $A_{2}$. Là encore, il existe de grandes variations des valeürs d'un jour sur l'autre. En moyenne, durant cette période, chez les renutris, l'AS de la lipase polentielle et de la phospholipase $A_{2}$ se sont pas modifiées par rapport aux témoins, celle de la lipase colipase dépendante est diminuée ( $-3,8$ fois).

L'ajout de méthionine au régime carencé montre en moyenne des AS diminuées de $-2,8$ fois pour la lipase potentielle, de - 9,4 fois pour la lipase colipase dépendante et augmentée de 2,2 fois pour la phospholipase $A_{2}$.

2) Débits (fig. 4). - Les trois débits sont stimulés après 3 jours de malnutrition ; ils montrent ensuite de grandes variations au cours de cette phase, mais là encore il n'y a pas d'altération progressivement croissante en fonction du temps de malnutrition. Les moyennes des débits sont peu différentes de celle des témoins pour les 3 enzymes pendant la malnutrition.

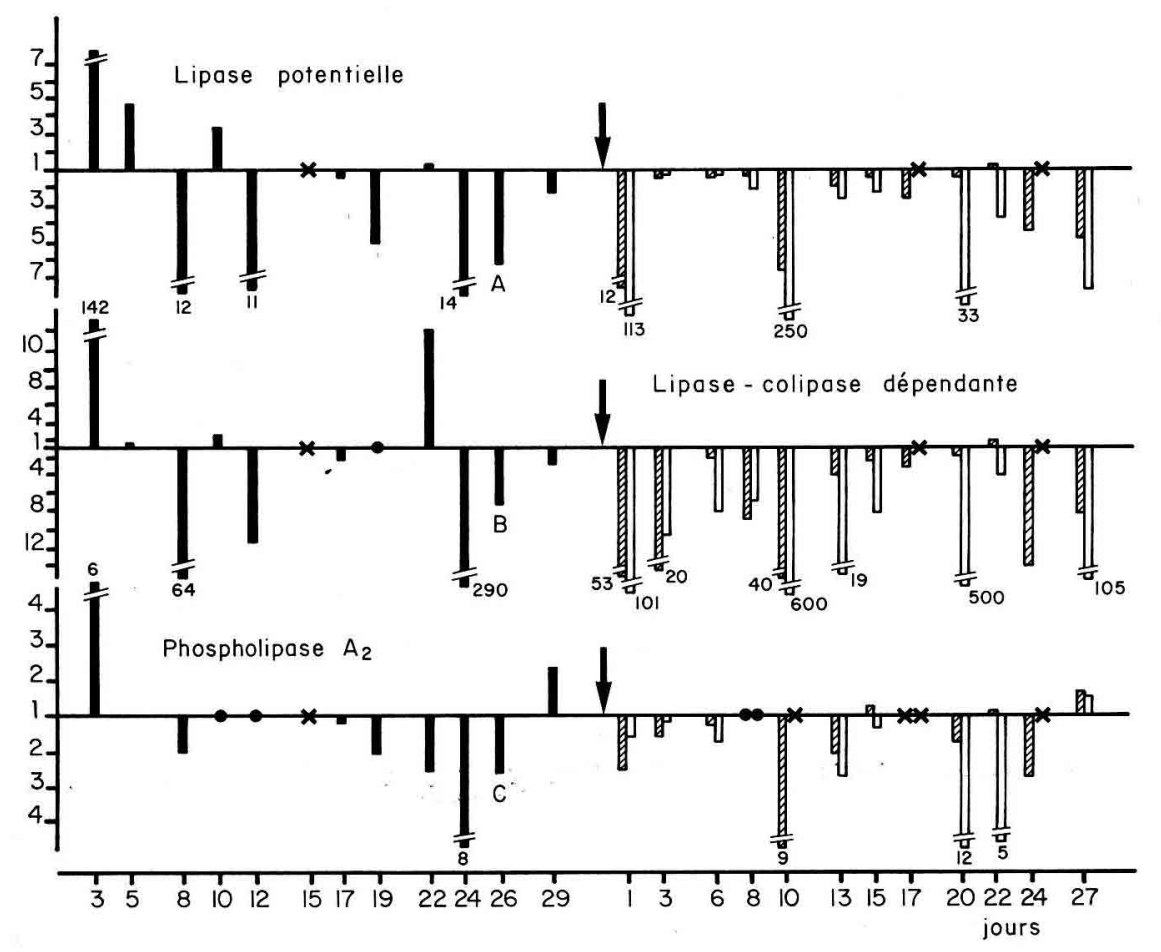

FIG. 4. - Débits de la lipase potentielle, de la lipase colipase dépendante et de la phospholipase $A_{3}$. Les débits sont obtenus en multipliant l'activité spécifique par le débit de protéines $(\mathrm{ml} / \mathrm{h})$. Légendes (voir fig. 3). 
Ces moyennes, durant la réalimentation, sont diminuées de 2,7 fois pour la lipase potentielle, de 11,2 fois pour la lipase colipase dépendante et de 2,4 fois pour la phospholipase $A_{2}$. Le simple ajout de méthionine au régime carencé aggrave la diminution des débits en moyenne ( -30 fois pour la lipase potentielle, - 100 fois pour la lipasecolipase dépendante ef seulement de $-1,6$ fois pour la phospholipase $A_{2}$ ).

\section{Discussion, conclusions.}

Les effets de la malnutrition protéique sur les activités enzymatiques digestives donnent, suivant les expériences, des résultats en apparence contradictoires. Ces contradictions peuvent être dues, non seulement au type de régime utilisé, sa durée d'administration, mais aussi au choix de l'espèce, son âge (jeune, adulte) ef aux différences individuelles.

Ce dernier fait a été bien observé au cours de notre expérimentation qui portait sur un grand nombre d'animaux. Les individus d'une même espèce présentent une sensibilité plus ou moins grande au stress de la malnutrition.

Les importantes variations constatées, au niveau du suc pancréatique et du pancréas, sont apparues aussi bien chez les animaux carencés et renutris que chez les témoins, sans que l'on puisse dire si elles sont dues essentiellement à des différences individuelles particulièrement importantes, non masquées par la rěunion des échantillons provenant de 3 rats, ou/ et à des rythmes endogènes non synchrones du nycthémère qui pourraient faire varier d'un dosage à l'autre les valeurs des activités.

Le régime de malnutrition que nous avons utilisé est carencé en protéines de mauvaise valeur biologique (gluten). Cette protéine est particulièrement pauvre en lysine. Le déficit protéique au niveau du régime est compensé par un apport d'amidon, de façon à maintenir les régimes isocaloriques. Donc, dans une certaine mesure, le régime hypoprotéique devient hyperglucidique. C'est un régime qui se rapproche de ceux consommés par l'Homme ef qui entraîne le syndrome du Kwashiorkor.

Le débit du suc pancréatique chez les carencés n'est pas altéré de façon continue eł régulière avec la durée de la malnutrition. Cependant, il est en général diminué. La chute du poids du pancréas semble être la cause première, dans la mesure où ce. débit devient voisin chez les deux groupes d'animaux lorsqu'on le rapporte à $100 \mathrm{~g}$ de poids corporel. La réalimentation équilibrée rehausse les débits de sucs chez les renutris.

Les débits de protéines suivent grossièrement les fluctuations des débits de suc, ce qui indique une certaine stabilité de la teneur en protéines des sucs pancréatiques.

L'ajout de méthionine stimule les débits de sucs et de protéines les premiers jours, mais cet effet est fugace.

Avant de discuter les effets de la malnutrition protéique, puis de la réalimentation sur les enzymes digestives, plusieurs rappels de portée générale sont à faire :

- Notre expérimentation a une certaine durée, entre le début et la fin, il s'est écoulé 2 mois. Les animaux, jeunes au début de l'expérience, sont adultes à la fin de celle-ci.

- Pour chaque prélèvement, les animaux sont sacrifiés, donc, d'un jour à l'autre, les dosages sont effectués sur des rats différents. 
- Les dosages enzymatiques sont effectués sur des animaux distincts pour le suc pancréatique et pour le pancréas.

Un résultat net et important qui ressort de notre travail est que la malnutrition, comme la renutrition, n'entraîne pas, à quelques exceptions près, de manifestations similaires pour toutes les enzymes. Comme pour un jour donné, les dosages ont lieu sur les mêmes pancréas ou les mêmes sucs, les différences individuelles ne jouent pas. Cela démontre que la malnutrition ef la réalimentation n'interviennent pas de la même façon sur toutes les enzymes, ce qui revient à dire que chaque enzyme ayant sa propre séquence de synthèse, celle-ci exprime une sensibilité différente vis-à-vis de la malnutrition et de la réalimentation.

Une autre constatation que nous faisons, c'est l'absence de synchronisme entre les valeurs trouvées dans le suc pancréatique et celles trouvées dans les extraits de pancréas. Une activité enzymatique peut diminuer dans le suc et augmenter dans la glande ou inversement, pour des temps de malnutrition et de réalimentation proches. Tandon ef al. (1969) avaient déjà mis en évidence cette désynchronisation entre le suc ef le pancréas au cours de la phase de récupération.

Nous trouvons aussi qu'en moyenne, les activités de la lipase-colipase dépendante sont plus altérées que celles de la lipase potentielle, ce qui signifierait que, proportionnellement, la colipase est plus sensible à la malnutrition que la lipase, ce qui sous-entend des voies de synthèse différentes pour l'enzyme et son cofacteur.

Un autre point que nous pouvons envisager est celui de la rétention des enzymes au cours de la malnutrition. Nous pouvons estimer grossièrement qu'il y a rétention accrue ou inhibition de l'excrétion lorsque l'activité spécifique d'une enzyme augmente dans le pancréas et diminue dans le suc et, qu'inversement, l'excrétion est accélérée lorsque l'activité spécifique diminue dans le pancréas et augmente dans le suc. On peut ainsi estimer que l'excrétion de la phospholipase $A_{2}$ est accrue ef que c'est plutôt l'inverse pour la lipase.

Les diminutions d'activités que l'on peut constater ne sont jamais progressives avec le temps de malnutrition. En particulier, cette phase ne s'achève pour aucune des enzymes par un écroulement de l'activité, au contraire. On peut donc estimer que l'organisme trouve encore à ce stade de malnutrition, grâce aux apporis endogène et exogène d'acides aminés, de quoi maintenir l'essentiel des activités digestives du pancréas. Il aurait été intéressant de prolonger au-delà le temps de malnutrition, car, par épuisement du pool endogène des acides aminés, l'effet $d u$ pool exogène devenant prédominant, l'impact de la malnutrition aurait pu apparaître plus nettement.

Lors de la réalimentation équilibrée, nous constatons comme Robberecht ef al. (1967), au début, une stimulation des activités spécifiques des enzymes en général. Mais, nous montrons de plus que cette stimulation n'existe pas pour toutes les enzymes et qu'elle est précédée (suc) ou suivie (pancréas) d'une importante inhibition. Ces effets sont généralement de grande amplitude et ils sont fugaces.

Les activités totales dans le pancréas et les débits dans le suc, malgré les déficits existants dans les quantités totales de protéines du pancréas et les débits de protéines du suc jusqu'à la fin de la réalimentation, montrent souvent un retour à la normale entre le $15^{\mathrm{e}}$ et le $21^{\mathrm{e}}$ jour, avec une nouvelle diminution en fin de phase de réalimentation. Cela est vrai, en particulier, pour les activités lipasique potentielle et colipasique dépendante. Il est à noter que le débit global de la lipase colipase dépendante 
est plus faible, au cours de cette phase, que celui de la lipase potentielle. La phospholipase $\mathrm{A}_{2}$ montre une excellente récupération dans le pancréas, un peu moins bonne dans le suc.

La supplémentation du régime carencé avec 0,6 p. 100 de méthionine n'entraîne pas d'amélioration significative pour les activités enzymatiques par rapport à ce que donnait la carence pure. Au niveau des activités totales du pancréas et des débits dans le suc, les valeurs trouvées montrent des altérations qui paraissent s'aggraver avec le temps. Il existe cependant une exception : l'activité spécifique de la lipase-colipase dépendante est améliorée dans le pancréas par cette supplémentation en méthionine. Ce résultat est encore un argument de plus en faveur d'une synthèse distincte entre la lipase et son cofacteur, la colipase.

Journées Ingestion-Digestion-Absorption de l'Association française de Nutrition, Paris, 15-16 novembre 1979.

\section{Références}

BARBEZAT G. O., 1967. The exocrine pancreas and protein calorie malnutrition. Afr. Med. J., 41, 84.

BELLEVILLE J., BOUZIANE M., PROST J., 1980. Influence d'un régime de malnutrition protéique ( 5 p. 100 de gluten) et d'une réalimentation équilibrée ( 15 p. 100 de caséine) sur les activités de la lipase potentielle, de la lipase-colipase dépendante et de la phospholipase $A_{2}$. I. Dans le pancréas de rat en croissance. Reprod. Nutr. Dévelop., 20, 1247-1255.

BELLEVILLE J., PROST J., GILLET M., 1978. Effets de régimes riches en triglycérides et en phospholipides sur les activités de la lipase ef de la phospholipase A du suc pancréatique ef du pancréas de rat. Arch. int. Physiol. Biochem., 86, 631-643.

LEMIRE S., IBER F. L., 1967. Pancreatic secretion in rats with protein malnutrition. Johns Hopkins med., 120, 21-25.

LYMAN R. L., WILCOX S. S., 1963. Effects of acute amino acid deficiencies on carcass composition and pancreatic function in the force-fed rat. I. - Deficiencies of histidine, methionine, phenylalanine and threonine. J. Nutr., 79, 28-36.

ROBBERECHT P., VANDERMEERS A., KHAYAT M. H., RATHE J., CHRISTOPHE J., 1967. Synthèse et excrétion des hydrolases pancréatiques chez le rat dont la malnutrition protéqque prend fin. Bull. Soc. Chim. biol., 49, 1735-1750.

TANDON B. N., GEORGE P. K., SAMA S. K., RAMACHANDRAN K., GANDHI P. C., 1969. Exocrine pancreatic function in protein calorie malnutrition disease of adults. Am. J. clin. Nutr., 22, 1476-1482.

TANDON B. N., GEORGE P. K., SAMA S. K., RAMACHANDRAN K., GANDHI P. C., 1970. Recovery of exocrine pancreatic function in adult protein calorie malnutrition. Gastroenterology, 60, $358-362$. 